Meta

Journal des traducteurs

Translators' Journal

\title{
La Bibbia Come Testo Letterario da Tradurre
}

\section{Carlo Buzzetti}

Volume 32, numéro 1, mars 1987

La traduction biblique

Bible Translation

URI : https://id.erudit.org/iderudit/002181ar

DOI : https://doi.org/10.7202/002181ar

Aller au sommaire du numéro

\section{Éditeur(s)}

Les Presses de l'Université de Montréal

\section{ISSN}

0026-0452 (imprimé)

1492-1421 (numérique)

\section{Découvrir la revue}

\section{Citer cet article}

Buzzetti, C. (1987). La Bibbia Come Testo Letterario da Tradurre. Meta, 32(1), 55-63. https://doi.org/10.7202/002181ar

\section{Résumé de l'article}

L'auteur se propose de mettre en évidence certains traits caractéristiques des textes littéraires et de montrer dans quelle mesure de tels textes sont traduisibles. Il envisage en particulier le cas de la traduction biblique. - Dans les deux premiers paragraphes, il analyse, d'une part, les principales fonctions du langage (fonctions descriptive, expressive et suggestive) et, d'autre part, la connotation par rapport à la dénotation. Or, ce qui confère à un texte sa valeur typiquement littéraire, c'est le rôle prédominant qu'y jouent les fonctions expressive et suggestive, alliées à la présence essentielle des connotations. Mais, affirmer cela, n'est-ce pas amener de l'eau au moulin de ceux qui prétendent que la traduction littéraire serait impossible ? En effet, comment rendre dans une traduction les caractéristiques esthétiques d'un texte, qui sont loin d'être saisies même par ceux qui le lisent dans sa version originale? L'auteur répond à cette objection en affirmant que s'il est exact que la communication absolue est un objectif inatteignable, il n'en reste pas moins qu'une réflexion linguistique sérieuse nous garde de tout scepticisme radical à cet égard. Si l'on prend le cas des connotations, il est vrai qu'on ne peut pas les rendre mot pour mot, ou même phrase par phrase. Toutefois, il convient de considérer les valeurs connotatives par rapport à un contexte plus général, ou à des unités de texte dépassant celles du mot ou de la phrase : à cet égard, le bon traducteur prouve sa compétence en étant capable de rendre le ton général du texte. De plus, il convient d'évaluer les divers aspects d'un texte littéraire cas pour cas, car les caractéristiques formelles n'ont pas toutes la même importance quant à l'impact général du message. C'est ce que l'auteur montre par quelques exemples bibliques (poèmes alphabétiques de l'Ancien Testament, épîtrespauliniennes, style johan-nique...). L'essentiel consiste à ne pas se lancer dans la traduction sans une réflexion théorique préalable, aussi bien linguistique qu'herméneutique. -En conclusion, l'auteur affirme : $1^{\circ}$ ) que la Bible appartient véritablement au domaine littéraire ; par conséquent, il serait illégitime de la traduire en négligeant sans cesse tous ses traits typiquement littéraires pour ne se préoccuper que des dénotations ; $2^{\circ}$ ) que la Bible n'est pas avant tout littérature ; par conséquent, il serait illégitime de la traduire en ne portant son attention que sur ses aspects esthétiques, sans se soucier de leur valeur par rapport à la foi, point central des récits bibliques.
Ce document est protégé par la loi sur le droit d'auteur. L’utilisation des services d’Érudit (y compris la reproduction) est assujettie à sa politique d'utilisation que vous pouvez consulter en ligne.

https://apropos.erudit.org/fr/usagers/politique-dutilisation/ 


\title{
LA BIBBIA COME TESTO LETTERARIO DA TRADURRE
}

\author{
Carlo BuzzetTI \\ Université salésienne, Rome, Italie
}

Résumé

L'auteur se propose de mettre en évidence certains traits caractéristiques des textes littéraires et de montrer dans quelle mesure de tels textes sont traduisibles. Il envisage en particulier le cas de la traduction biblique. - Dans les deux premiers paragraphes, il analyse, d'une part, les principales fonctions du langage (fonctions descriptive, expressive et suggestive) et, d'autre part, la connotation par rapport à la dénotation. Or, ce qui confère à un texte sa valeur typiquement littéraire, c'est le rôle prédominant qu'y jouent les fonctions expressive et suggestive, alliées à la présence essentielle des connotations. Mais, affirmer cela, n'est-ce pas amener de l'eau au moulin de ceux qui prétendent que la traduction littéraire serait impossible? En effet, comment rendre dans une traduction les caractéristiques esthétiques d'un texte, qui sont loin d'être saisies même par ceux qui le lisent dans sa version originale? L'auteur répond à cette objection en affirmant que s'il est exact que la communication absolue est un objectif inatteignable, il n'en reste pas moins qu'une réflexion linguistique sérieuse nous garde de tout scepticisme radical à cet égard. Si l'on prend le cas des connotations, il est vrai qu'on ne peut pas les rendre mot pour mot, ou même phrase par phrase. Toutefois, il convient de considérer les valeurs connotatives par rapport à un contexte plus général, ou à des unités de texte dépassant celles du mot ou de la phrase : à cet égard, le bon traducteur prouve sa compétence en étant capable de rendre le ton général du texte. De plus, il convient d'évaluer les divers aspects d'un texte littéraire cas pour cas, car les caractéristiques formelles n'ont pas toutes la même importance quant à l'impact général du message. C'est ce que l'auteur montre par quelques exemples bibliques (poèmes alphabétiques de l'Ancien Testament, épîtres pauliniennes, style johannique...). L'essentiel consiste à ne pas se lancer dans la traduction sans une réflexion théorique préalable, aussi bien linguistique qu'herméneutique. - En conclusion, l'auteur affirme: $\left.1^{\circ}\right)$ que la Bible appartient véritablement au domaine littéraire; par conséquent, il serait illégitime de la traduire en négligeant sans cesse tous ses traits typiquement littéraires pour ne se préoccuper que des dénotations ; $2^{\circ}$ ) que la Bible n'est pas avant tout littérature ; par conséquent, il serait illégitime de la traduire en ne portant son attention que sur ses aspects esthétiques, sans se soucier de leur valeur par rapport à la foi, point central des récits bibliques.

La Bibbia è un libro, un testo. A volte, in determinate condizioni linguistiche, essa non può essere compresa se non a patto di essere tradotta. Ma se la Bibbia è un testo letterario, la sua traduzione non è un rischio grave ? Si dice spesso che le opere letterarie sono talmente identiche con la lore forma originale che il tradurre implica deformazioni inevitabili (questo è detto soprattutto per la poesia...). Qui tentiamo di individuare un aspetto tipico dei testi letterari e indichiamo in che misura essi si possono tradurre. Il tutto, ovviamente, con particolare riferimento alla Bibbia. 


\section{LE DIVERSE FUNZIONI LINGUISTICHE}

Il linguaggio è uno strumento utilizzabile per vari scopi. Uno schema semplice, riconosciuto da molti linguisti, filosofi e teologi ${ }^{1}$, indica tre funzioni principali : descrittiva, espressiva e suggestiva. Quando si parla-scrive, sono coinvolti sempre tre protagonisti : il soggetto-fonte, l'argomento, il soggetto-recettore. Se chi parla-scrive concentra l'attenzione su un argomento estraneo a lui e al recettore, il linguaggio assume un ruolo descrittivo, informativo; usa in prevalenza la "terza persona" grammaticale; si avvicina ai modi del linguaggio scientifico. Se chi parla-scrive fa di se stesso l'argomento, allora il linguaggio svolge una funzione espressiva : manifesta l'interiorità, diventa una revelazione e uno sfogo. Se chi parla-scrive ha in mente soprattutto il recettore, il linguaggio funziona come appello, pressione, suggestione, stimolo, incitamento : non serve tanto a far sapere quanto a far agire : è il regno della "seconda persona" e dell'imperativo ${ }^{2}$.

Le tre funzioni sono spesso intrecciate e difficili da separare. Ma rimane vero che la prima si svolge prevalentemente nei testi scientifici, la seconda in quelli lirici, la terza in quelli parenetici o normativi ${ }^{3}$.

Non è facile trovare una definizione di ciò che rende "letterario" un testo 4 ; tuttavia nella nostra prospettiva è possibile affermare che in esso dominano le funzioni espressiva e suggestiva : le parole vengono usate soprattutto come sintomi rivelatori di sentimenti e come segnali per provocare pensieri e azioni.

\section{DENOTAZIONE E CONNOTAZIONE}

Da un altro punto di vista la riflessione sul linguaggio ha condotto a conclusioni differenti, ma per noi analoghe. Infatti per secoli la linguistica tradizionale aveva trascurato gli elementi non strettamente logici del discorse e, senza negarli, li aveva lasciati alla competenza della critica letteraria ; così, si diceva, l'arte rimaneva oggetto di valutazione esclusivamente artistica... $\mathrm{E}^{\prime}$ interessante notare che i traduttori furono sempre consapevoli della presenza di elementi non soltanto logici : sfumature, colorazioni, toni, stili... sfuggivano alla presa dell'analisi filologica tradizionale, eppure dovevano essere tradotti perchè la traduzione si potesse dire un buon equivalente, fedele. Che fare? Di fatto, si affermò in molte maniere che soltanto un poeta poteva tradurre bene un poeta, perchè solamente un tale dono poteva produrre qualcosa di equivalente. Lo stesso fu applicato alla letteratura in genere; si diceva che l'arte letteraria contiene elementi indefinibili, ma decisivi, $i$ quali in una traduzione non eccezionale sono fatalmente destinati ad essere deformati. Al limite, si pensava (e molti pensano ancora oggi) che un'opera letteraria non è mai veramente traducibile.

Studi linguistici più recenti ${ }^{5}$ hanno prestato maggiore attenzione agli aspetti non immediatamente logici del linguaggio (all'inizio si parlava di "sensi affettivi delle parole"). Il linguista più famoso in tale ambito è l'americano Leonard Bloomfield (1887-1949); egli introduce una distinzione oggi molto diffusa (benchè a volte contestata) : ogni termine posssiede una "denotazione" e una "connotazione" ; con la prima egli indica "il minimum dei tratti oggettivi comuni grazie ai quali si può definire tale termine per tutti i parlanti"; con la seconda "tutti gli altri tratti distintivi del significato che possono essere o non essere aggiunti alla denotazione, essere o no percepiti dall'ascoltatore, essere voluti o non esserlo nell'atto della comunicazione"6. Oggi psicologi, filosofi e linguisti sono ampiamente concordi nel sostenere idee riconducibili a quelle : ogni atto di linguaggio, anche se sembra del tutto "denotativo" (cioè descrittivo e oggettivante), può contenere preziosi e reconditi elementi "connotativi" che dicono qualcosa del soggetto-fonte o del soggetto-recettore.

Come si realizzano le "connotazioni" ? A vari livelli : a) senza dubbio negli aspetti mimico-gestuali che accompagnano la parola ; b) ovviamente sul piano fonetico del 
linguaggio parlato (pensiamo alla ricca gamma di risorse cui fa appello l'arte oratoria, la prassi della recitazione...) ; c) a livello di testo scritto : è vero che la scrittura si è perfezionata soprattutto per esprimere le "denotazioni" (e quindi le funzioni informative) cosiò che l'interpretazione di un testo scritto è sempre anche un trovare $\mathrm{e}$ un esprimere le connotazioni nascoste; tuttavia alcune di esse sono direttamente espresse attraverso fenomeni linguistici formali evidenti. Elenchiamo qui di seguito ambiti che non sono identici con le dimensioni mimiche o fonetiche del linguaggio.

1. Già l'insieme della punteggiatura (il segno esclamativo e interrogativo, i punti, le virgole...) e l'impiego delle varie risorse tipografiche (il grassetto, il corsivo, il maiuscolo, le spaziature...) hanno proprio la funzione di suggerire sfumature di significato secondarie rispetto a quelle principali delle parole : il loro uso e la loro comprensione sono perciò diretti verso le "connotazioni".

2. Anche le differenze lessicali possono diventare strumento di diverse connotazioni. In ogni lingua esistono termini sinonimi dal punto di vista denotativo, ma distinti proprio per la carica connotativa. Ad esempio, le tre espressioni : "egli è mio padre" ; "egli è mio genitore" ; "egli è il mio papà" possono essere considerate equivalenti dal punto di vista logico perchè indicano la medesima persona; ma - innegabilmente - "padre", "genitore" e "papà" possiedono 'sfumature' di significato ben diverse, comunicano sentimenti e connotazioni non identiche! Gli esempi si possono moltiplicare. Cosiò, dicendo "la mia casetta" a volte non si vogliono indicare le piccole dimensioni oggettive di un'abitazione ma, eventualmente, sentimenti di amore e nostalgia ; in assenza di essi, la medesima realtà potrebbe essere detta semplicemente "casa". Oppure : la differenza che esiste tra un termine generico e uno specifico, non è solamente logica e denotativa : può servire anche a indicare particolari connotazioni ; pensiamo, ad esempio, al diverso significato che in un contesto polemico possono assumere frasi come queste : "se vuoi, prenderò quel vestito !" oppure "se vuoi, prenderò quel pezzo di stoffa !".

3. Il contesto esistenziale dell'uso di un termine è un ambito che supere quello linguistico; lo prendiamo in considerazione perchè di fatto è il luogo e il mezzo che fa sorgere certe connotazioni. Cosiò, che cosa significa il termine "fucile" ? Esiste un significato denotativo piuttosto stabile e comune; ma le connotazioni variano sensibilmente in tempo di pace o in condizioni di guerra, per uno sportivo o per un militare, per un assassino o per una vittima, per un fabbricante d'armi o per un pacifista, per un meccanico o per un artista... Che cosa significa "pecora" per un pastore che trascorre l'esistenza quasi sempre vicino al suo gregge, oppure per un cittadino che lavora in un lanificio ? Sicuramente la parabola evangelica del buon pastore che ama le sue pecore... ha risonanze differenti per i due tipi di recettori.

E cosiò di seguito. Per noi può essere utile osservare che il linguaggio letterario è definito anche in base alla massiccia presenza di connotazioni ${ }^{7}$. E' cosiò possibile fare un facile parallelo : la predominanza di alcune funzioni (espressiva e suggestiva) corrisponde alla presenza notevole di alcuni aspetti (le connotazioni) per stabilire la fisionomia tipica della letterarietà di un testo.

\section{UN TESTO LETTERARIO SI PUÒ TRADURRE?}

Certi filosofi e linguisti hanno accolto osservazioni simili a quelle precedenti per giungere poi a conclusioni negative e ‘disperate' circa la possibilità di comunicare. Si è detto : a proposito della realtà, ogni persona possiede prospettive radicalmente sue, incomparabili rispetto a quelle di altri ; ogni situazione è unica, diversa dalle precedenti e da quelle successive; perciò le parole, per quanto stabili e uguali, di fatto possiedono significati sempre diversi. La comprensione è illusoria ; in realtà essa non si realizza mai veramente, perchè in nessun caso le parole trasmettono il medesimo carico di 
connotazioni per chi parla-scrive e per chi riceve il messaggio. Una prova evidente di questa radicale incomunicabilità si ha soprattutto in occasione di testi ad alto contenuto artistico : qui la presenza di connotazioni è tanto notevole da rendere impossibile un accordo interpretativo ; effettivamente le interpretazioni si moltiplicano e spesso sono persino contrarie... Di conseguenza sembra più onesto affermare che il linguaggio si limita a produrre illusori frammenti di comunicazione, ma non trasmette mai con fedeltà un pensiero.

Dal nostro punto di vista notiamo che questo modo di giudicare implica una condanna radicale anche circa qualsiasi traduzione : infatti tradurre è impossibile, è un gesto destinato a mutare e quindi stravolgere le connotazioni, le caratteristiche esteticoletterarie di un testo, la sua assoluta unicità ; se non si può veramente comunicare, a maggior ragione non si può tradurre, dal momente che la traduzione si fonda su una duplice comunicazione (dal testo-fonte al recettore-traduttore; da questi al nuovo destinatario). Citiamo una sintesi autorevole : "Quando si dice che la traduzione è impossibile, nove volte su dieci si pensa a queste connotazioni che mettono in questione non solamente la possibilità di passaggio da civiltà a civiltà, da "visione del mondo" a "visione del mondo", da lingua a lingua, ma, in fondo, da individuo a individuo persino all'interno di una civiltà, di una 'visione del mondo', di una lingua che sono comuni. In fin dei conti la nozione di connotazione pone alla teoria della traduzione sia il problema della possibilità sia quello dei limiti della comunicazione interpersonale intra-soggettiva"8.

Lo ripetiamo, questa sfiducia si rivolge soprattutto ai testi letterari perchè carichi di valori connotativi. Ma ora è il momento di introdurre una distinzione. E' vero che non pochi letterati (e filosofi) moderni affermano l'insuperabile solitudine di ciascuno, ricordano il tormento di non trovare parole per farsi comprendere e il tormentoso sospetto di non essere compresi anche quando l'altro dice di aver capito. Ma la loro posizione, mentre fa leva su un'esperienza innegabile (alcuni aspetti soggettivi dei significati, alcune sfumature sono davvero difficili da comunicare) poi si allarga a conclusioni generali molto poco motivate (= ogni comunicazione è sempre illusoria...). Forse quei moderni sviluppano una implicita polemica contro chi, nel passato, ha sempre presupposto la facile adeguatezza tra mondo interiore e strumenti linguistici, la fondamentale identità delle varie situazioni umane : e per polemica — appunto enfatizzano il contrario. Tuttavia essi finiscono con il mitizzare una ideale "vera comunicazione' opponendola alla comunicazione reale che essi svalutano. Ebbene : la comunicazione che concretamente si realizza, possiede dei limiti evidenti, ma non per questo non è vera. I moderni linguisti rigorosi hanno superato una certa tradizionale superficialità, non si lasciano ipnotizzare nè dai successi nè dalle difficoltà del comunicare ; non accolgono l'idea, appassionatamente diffusa da certi letterati, secondo cui la comunicazione è perfetta o inesistente; al contrario, essa si realizza a livelli e in forme differenti, più o meno soddisfacenti secondo $i$ vari punti di vista. La comunicazione totale, assoluta e senza modifiche, è un miraggio che non deve allontanarci dalla realtà. Un buon linguista sa bene che le situazioni sono moltissime (potenzialmente infinite) mentre le parole, i termini e le loro combinazioni sono relativamente poche; di conseguenza vi sono sempre parole ed espressioni identiche che si riferiscono a realtà per qualche aspetto diverse; tuttavia la diversità non è mai assoluta ; anzi, essa si accompagna e si combina sempre con una reale somiglianza...

Quindi una seria riflessione linguistica porta a respingere ogni scetticismo radicale. Quando colui che parla-scrive usa la medesima parola per due o più realtà distinte, non necessariamente pensa o vuol fa pensare che esse sono del tutto identiche : piuttosto, egli pensa e vuol far pensare che sono molto simili. Il linguaggio esprime le 
caratteristiche maggiori, non tutte le sfumature ; in termini tecnici si parla di aspetti macroscopici o microscopici, tratti semanticamente pertinenti o non pertinent ${ }^{9}$, sociali o individuali... : il linguaggio raccoglie ed esprime quegli aspetti del mondo - compreso il mondo dei sentimenti - che sono accessibili a molti. Cosí quando io dico-scrivo "ho visto una bella casa" chi ascolta-legge capisce, ma sempre e soltano entro certi limiti ; ad esempio, non può sapere che io mi referisco a una casa gialla con le persiane verdi, se io non lo dico ; a maggior ragione, non avvertirà quali suggestioni o ricordi o sentimenti quella casa provoca in me, a meno che io intraprenda la fatica di esprimere anche quegli aspetti. Però, a grandi linee, egli può comprendere davvero : la mia frase gli offre i tratti pertinenti che gli permettono di dire : so di che cosa tu parli. Egli non comprende tutto, non tutti i minimi particolari, perchè la mia esperienza è, al limite, unica e quindi irripetibile e quindi incomunicabile : ma la comunicazione può essere sempre arricchita, perfezionata e migliorata; la comprensione è suscettibile di progresso. Ciò che è parziale, non per questo è falso e illusorio.

\section{TRADUZIONE DELLE CONNOTAZIONI}

Anche i sentimenti, anche le connotazioni hanno un certo grado di socialità ; quindi si possono tradurre, almeno per approssimazioni successive. E' vero che, ad esempio, ogni uditore-lettore comprende diversamente il termine "pecora" ; ma è anche vero che un moderno cittadino può comprendere l'antica parabola di un pastore che chiama per nome le sue pecore... ; infatti egli può capire che in quel contesto la "pecora" evoca sentimenti simili a quelli che in lui, forse, evoca il termine "gatto"; ha esperienza del "chiamare per nome", del "seguire", del "dare la vita".. Non giungerà forse mai a comprendere in tute le sfumature il rapporto pastore-pecora; ma non per questo la sua comprensione è falsa.

Come si traducono le connotazioni ? Molte si riproducono automaticamente mediante l'intero contesto, quindi il traduttore non deve preoccuparsene in maniera speciale. Naturalmente il contesto è sconfinato : il brano, l'intera opera, l'epoca, la letteratura... per questo la traduzione delle connotazioni non è mai compiuta definitivamente. Le connotazioni più tipicamente letterarie sono proprio di natura contestuale, sono legate a livelli di linguaggio, generi e stili, difficilmente definibili da chi non possiede una esercitata sensibilità letteraria. Il traduttore deve essere in grado di percepire e di riprodurre un livello tecnico o familiare o ricercato, un genere lirico o narrativo o epico, uno stile ordinario o prezioso o brillante... Non sempre è facile "tradurre" tutto ciò mediante una semplice traduzione parola per parola; le connotazioni, infatti, spesso risultano da un insieme che non si può intendere come somma materiale delle parti. Così l'abilità del buon traduttore si manifesta anche nel saper mettere in pratica un criterio che noi diremo "dell'equilibrio" o "del compenso". Consideriamo, ad esempio, un brano nel quale domina un generale tono volgare, espresso soprattutto da alcuni termini 'volgari'; se il traduttore non può trovare un buon equivalente 'volgare' per ciascuno di essi, non per questo deve dimenticare l'insieme; anzi, egli può e deve ricreare il tono complessivo del brano originale verificando la 'volgarità' globale. Lo stesso vale per molte altre caratteristiche letterarie : se non è possibile tradurle parola per parola (il che è molto frequente) si riproducono mediante equivalenze che, globalmente, rendono il brano tradotto simile al primo. La traduzione letteraria non è rivolta soprattutto a singole parole o singole frasi, ma si impegna a capire e far capire unità linguistiche più ampie. A livello di parole e di frasi sono spesso inevitabili le deformazioni e le "infedelà" ; ma il criterio del compenso aiuta a ristabilire l'equilibrio all'interno di una pagina. Per esempio : pensiamo di dover tradurre "gli saltò la mosca al naso" in una lingua che di fatto non possiede un'espressione idiomatica tanto colorita e dal significato corrispondente ; può darsi che 
il traduttore si veda costretto a usare una frase del tipo "egli si sdegnò ; ma non appena gli è possibile, egli userà un'espressione colorita per rendere una frase più smorta dell'originale; in tal modo l'equilibrio è rispettato, l'insieme delle connotazioni è fedelmente riprodotto.

\section{ESEMPI BIBLICI}

Almeno in un senso generale, la Bibbia è un testo "letterario" perchè in essa trovano spazio notevole le funzioni espressiva e impressiva, connotazioni di vario genere. Ma dobbiamo chiederci se la letterarietà della Bibbia sia centrale o accessoria ; infatti - al limite - anche un testo di botanica può raggiungere livelli molto artistici, eppure la funzione informativa rimane per esso primaria e questa, ovviamente, deve essere soprattutto curata in una traduzione; e per la Bibbia? E' molto diffusa una concezione che intende la Bibbia come un complesso di verità rivelate, indipendente 0 quasi dal modo con cui esse vengono presentate ${ }^{10}$; tale idea si riflette direttamente nelle traduzioni che mostrano di non dare importanza ad aspetti letterari anche macroscopici (per es. stampano come prosa pagine la cui natura è sicuramente poetica!).

Quella prospettiva è ingenua, illegittima e provoca distorsioni anche gravi ; tuttavia bisogna ammettere che, caso per caso, non è facile stabilire quanto importante sia la forma letteraria. La via migliore rimane quella di stabilire volta a volta se e quanto la forma sia un elemento decisivo per il contenuto stesso. E' noto che forma e contenuto sono per certi aspetti separabili e per altro verso molto connessi ${ }^{11}$; di conseguenza ogni caso deve essere valutato con cura. Vediamo qui alcuni esempi rappresentativi.

a) Non è raro incontrare nei testi poetici della Bibbia ebraica quella particolarità letteraria che è detta "acrostico alfabetico" (essa consiste nel fare in modo che ogni verso oppure ogni strofa inizi con una lettera dell'alfabeto, secondo l'ordine tradizionale) : si vedano le 22 strofe di Lamentazioni 3 ; quelle del Salmo 119... L'artificio è evidente ; il valore artistico è discutibile; l'aspetto letterario è comunque innegabile. Bisogna riprodurle in una traduzione? Di fatto quasi nessuno lo fa ; forse perchè non è facile realizzarlo senza gravi distorsioni del significato ; forse anche perchè i traduttori non lo giudicano importante ai fini della buona comprensione : lo considerano una forma poco o nulla legata al contenuto, sintomo di gusti letterari che non influiscono sul messaggio. Tuttavia trascurarlo del tutto non si può : un esegeta deve tener conto che le parole in ordine alfabetico sono state scelte anche per seguire una legge letteraria e quindi non sempre sono il mezzo più adeguato per esprimere il pensiero o il sentimento dell'autore ; come l'esegeta, anche il traduttore deve comprendere un brano alla luce del contesto e riprodurre ciò che gli sembra più importante; forse deve trovare la maniera di fornire al nuovo lettore un'informazione equivalente... 12.

b) Lo stile di Paolo è spesso complesso, a volte pesante e contorto. Sembra di poterlo considerare un riflesso della sua personalità forte e immediate, del suo pensiero rapido e appassionato. Concretamente, però, la sua forma costituisce anche un ostacolo per la comprensione, pare che già tra i primi lettori fosse diffuso un simile imbarazzo ${ }^{13}$. Per noi basta leggere il testo greco o il testo di qualche traduzione che si propone di imitare le caratteristiche dell'originale : spesso incontriamo brani nei quali è arduo seguire il filo dei pensieri. Un esame delle traduzioni mostra un fenomeno interessante : nel passaggio alla nuova lingua, sempre si opera un certo alleggerimento di quello stile (qualche participio è reso con forme verbali finite; oppure un sostantivo astratto diventa un verbo ; oppure una frase troppo lunga è spezzata in due...) Nessuno opera in senso contrario, rendendo lo stile più complesso e difficile; perchè ? Non è facile rispondere, ma osiamo suggerire un probabile motivo : più o meno esplicitamente, gli esegeti-traduttori pensano che l'elemento formale dello stile contorto non è sempre importante per il significato ; anzi, per favorire la comprensione del significato stesso si può e si deve ope- 
rare qualche modifica. Nessuno o quasi, tuttavia, sostiene l'opportunità di modificazioni tali da suggerire ai nuovi lettori l'idea che Paolo si esprime con frasi sempre brevi e lucide; questo sarebbe un tradimento che può influire sulla genuina comprensione del messaggio.

c) Alcune pagine del N.T. possiedono lo stile monotono dell'elenco : cosiò Apocalisse 7, quando presenta i 140000 eletti, ripete dodici volte il numero 12000 ; cosiò Matteo 1 , scrivendo la genealogia di Gesù, riprende sistematicamente l'ultimo nome citato e le medesime formule... Uno scrittore moderno eviterebbe simili pesantezze. Eppure la maggior parte delle traduzioni non modifica quello stile. Perchè ? Forse perchè si avverte che qui il "modo" è espressione della specifica natura del testo ; un'informazione circa il numero e le persone non è fedele se non trasmette anche l'idea che qui si propone qualcosa come un documento giuridico. Eventuali modifiche sono opportune o legittime a patto di comunicare un'impressione equivalente, cioè far capire che qui si tratta di un quasi-documento.

d) Gli studiosi sono unanimi nel riconoscere che gli scritti giovannei possiedono uno stile inconfondibile (per esempio : l'uso e la ripetizione di pochi vocaboli e pochi temi, generalmente originali ; una solennità ritmica che rende a volte ardua la distinzione tra prosa e poesia). Le traduzioni, tuttavia, non si comportano allo stesso modo di fronte a quelle particolarità stilistiche : mentre tutte tendono a riprodurre la terminologia tipica usando parole fisse, ben poche danno rilievo al ritmo di tanti brani e semplicemente li presentano come prosa comune ${ }^{14}$. Eppure quest'ultimo atteggiamento pare da giudicare come un difetto, anche se molto diffuso ; infatti, capire e far capire la presenza di una struttura ritmica non è irrilevante per la percezione del significato. Le parole hanno un peso che dipende anche dalla loro posizione nel brano, dai collegamenti, dalle ripetizioni... Di conseguenza sembra consigliabile non trascurare la possibilità di tradurre importanti aspetti del messaggio proprio mediante la scelta delle forme letterarie ${ }^{15}$. Naturalmente una traduzione non dovrebbe rendere la struttura più esplicita e più evidente di quanto essa lo sia nel testo originale ${ }^{16}$; ma non dovrebbe nemmeno eliminarla e, anzi, nei limiti del possibile, almeno in alcuni casi deve fare in modo che il lettore la possa individuare.

Gli esempi potrebbero essere moltiplicati. E' importante un'osservazione complessiva : gli aspetti letterari, anche evidenti, di un testo come la Bibbia in alcuni casi si riflettono scrupolosamente in una versione, mentre in altri casi sono del tutto eliminati o deformati. Non pare che all'origine sia da riscontrare una chiara teoria : a volte prevale un atteggiamento letteralista che automaticamente fa riprodurre certi elementi formali (ma ne distrugge altri); a volte domina la vaga convinzione che la forma sia rilevante per il contenuto; a volte si pensa che un aspetto stilistico sia del tutto separabile dal significato. Mai o quasi mai una traduzione è preceduta da una riflessione generale sul che cosa si traduce, a chi e con quale finalità ${ }^{17}$. Gli esempi considerati, e altri che possono essere discussi, mostrano l'opportunità o addirittura l'urgenza di un simile approccio teorico, generale, linguistico ed ermeneutico insieme.

\section{UNA PROSPETTIVA}

Suggeriamo una formula complessiva. E' vero che la Bibbia è un testo letterario : per l'abbondante presenza delle funzioni espressiva e suggestiva, per il ruolo notevole delle connotazioni, per l'innegabile influsso di vari modelli artistici, la Bibbia deve essere tradotta come si traduce una realtà letteraria vera e complessa. Anzi, la Bibbia si presenta non come un prodotto letterario, ma come una raccolta di scritti diversi per lingua, autore, epoca, genere, stile... Anche questa diversità letteraria esige di essere, in qualche misura e in qualche modo, rispettata e riprodotta. Tuttavia - e questo puó essere l'osservazione conclusiva - la Bibbia non è solamente letteratura, nè un 
accostamento di realtà letterarie disparate ; all'interno della sua netta varietà esiste una particolare unità, una dimensione che trascende la dimensione letteraria : la Bibbia è il ricordo scritto di una fede. Tale testimonianza di fatto ha assunto varie forme, sicuramente letterarie; eppure la Bibbia non è nata e non è stata accolta nè usata soprattuto come libro di arte linguistica : principale è sempre il suo riferimento alla storia di una fede. Di conseguenza, i vari aspetti letterari sono relativi alla fede; essi sono importanti nelle misura in cui la esprimono; anche la traduzione è dunque impegnata a riprodurli in quanto ne sono strumenti. Accennando di nuovo agli esempi visti sopra, si può dire : a) la forma dell'acrostico alfabetico indica quasi esclusivamente uno stile e una convenzione; essa non pare molto significativa della fede; per questo è trascurabile a vantaggio del significato ; b) lo stile di Paolo indica la passione esemplare con cui egli vive la fede ; uno stile più semplice, meno faticoso del suo, è legittimo in una versione purchè sappia esprimere quella passione; c) gli elenchi sembrano indicare fortemente $\mathrm{i}$ legami storico-giuridici tra certi fatti e certi aspetti della fede; una traduzione puó attenuare la loro monotonia soltanto se non distrugge la loro funzione primaria; d) lo stile giovanneo e altri stili analoghi presenti nella Bibbia sono innegabilmente "suggestivi" nel senso che danno solennità ieratica a racconti o discorsi ; essi ricordano, indirettamente ma realmente, la presenza gloriosa di Dio in avvenimenti e persone di questo mondo ; perciò la traduzione non deve banalizzarli con uno stile che si limita ad essere corretto ; a volte dovrà fare la scelta di utilizzare una forma semipoetica...

Il nostro abbozzo di regola generale rimane ancora generico : esige un'applicazione attenta e sfumata ; tuttavia ci pare utile almeno nel senso che dice : 1) la Bibbia è vera letteratura; quindi non è legittimo tradurla trascurando sempre i valori letterari, a favore di un appiattimento provocato in nome del primato assoluto delle denotazioni ; 2) la Bibbia non è soprattutto letteratura ; quindi non è legittimo tradurla mediante un'attenzione prioritariamente rivolta ai valori estetico-artistici senza valutare il lore significato dal punto di vista della fede. Evidentemente, tradurre la Bibbia implica un intelligente interpretare ${ }^{18}$.

Notes

1. Lo si fa risalire a K. Buehler (1934) : Sprachtheorie, Jena ; ̀̀ ripreso da vari autori : cf. B. Malmberg (1966) : les Nouvelles tendances de la linguistique, Paris ; M. Black (1968) : The Labyrinth of Language, London ; E. Nida (1964) : Toward a Science of Translating, Leiden; etc.

2. Altri autori (per es. Alonso Schökel $1966:$ La parola ispirata, Brescia) indicano quelle tre funzioni con termini diversi : simbolo, sintomo, segnale.

3. Un esempio semplice e illuminante è offerto da B. Malmberg, op. cit., pp. 308-309, a commento dell'esclamazione "Piove !" Circa l'intreccio delle varie funzioni nel dialogo, cf. Alonso Schökel, op. cit., p. 119.

4. Cf. lo studio ormai classico di R. Wellek - A. Warren (1942) : Theory of Literature, New York, parte I ; cf. anche V. Coletti (1978) : Il linguaggio letterario, Bologna.

5. G. Mounin (1963) : les Problèmes théoriques de la traduction, Paris, cita a questo proposito i contributi di Charles Bally, un discepolo di F. de Saussure, e di Edward Sapir.

6. Cosi in G. Mounin (1965) : Teoria e storia della traduzione, Torino, p. 105.

7. Cf. Wellek - A. Warren (1963) : Theory of Literature, New York, cap. II, oppone il linguaggio scientifico "tipicamente denotativo" al linguaggio letterario "principalmente connotativo".

8. G. Mounin : les Problèmes théoriques de la traduction, p. 168.

9. Cf. E. Sapir (1969) : Il linguaggia, Torino, pp. 12-13.

10. Cf. L. Alonso Schökel : op. cit., pp. 138-139 : egli polemizza verso una simile maniera di comprendere e presentare la Bibbia.

11. Su tale tema si legga : U. Eco (1971) : Le forme del contenuto, Milano.

12. Mi sembra da notare il buon equivalente trovato da alcune versioni recenti : la spagnola (Dios habla hoy) $\mathrm{e}$ la tedesca (Die Gute Nachricht) che usano dei numeri successivi per indicare le strofe del Salmo 119 . Si veda anche la notazione francese (la Bible en français courant) a Lamentazioni 3.

13. Cf. 2 Pietro 3, 16. 
14. Una notevole eccezione in tal senso è costituita dalla Bible de Jérusalem dove D. Mollat, curatore del vangelo di Giovanni, ha scelto la forma poetica per molti discorsi di Gesù ; in tal modo il traduttore è sulla linea di opinioni esegetiche autorevoli (cf. D. M. Smith 1965: The Composition and Order of the Fourth Gospel, Yale, e gli altri autori da lui citati, tra cui R. Bultmann).

15. Un esempio molto concreto : durante la versione italiana in lingua corrente dell'A.T., si è giunti alla conclusione di presentare in forma vistosamente poetica l'inizio di Genesi perchè così il lettore è aiutato a comprendere più fedelmente il significato dell'originale ebraico.

16. Il compito di esplicitare la struttura è esegetico-didattico : si veda A. Vanhoye (1963) : la Structure littéraire de l'épitre aux Hébreux, Paris ; de Waard-Smalley (1979) : A Translator's Handbook on the Book of Amos, New York (infatti, le United Bible Societies danno spazio allo studio della struttura proprio nei più recenti "Helps for Translators").

17. Ricordiamo alcuni prodotti recenti della linguistica del tradurre applicata alla Bibbia : C. Buzzetti (1973) : La Parola Tradotta, Brescia, soprattutto pp. 147-156 (esiste anche l'edizione spagnola : Traducir la Palabra, Estella, 1976) ; l'ottimo libro di J.C. Margot (1979) : Traduire sans trahir, Lausanne.

18. Uno sviluppo ulteriore di questa conclusione si può leggere in un nostro saggio pubblicato da Rivista Biblica, Brescia, XXII (1974) 